\title{
Is copeptin a new potential biomarker of insulin resistance in polycystic ovary syndrome?
}

\author{
Justyna Widecka, ${ }^{1,2}$, Katarzyna Ozegowska3 ${ }^{3}$, Beata Banaszewska ${ }^{3}$, Anna Kazienko4, \\ Krzysztof Safranow ${ }^{1}$, Dorota Branecka-Wozniak ${ }^{1}$, Leszek Pawelczyk ${ }^{3}$, Rafal Kurzawa ${ }^{1,2}$ \\ ${ }^{1}$ Pomeranian Medical University in Szczecin, Poland \\ ${ }^{2}$ The Fertility Partnership, Vitrolive Fertility Clinic, Szczecin, Poland \\ ${ }^{3}$ Karol Marcinkowski University of Medical Sciences in Poznan, Poland \\ ${ }^{4} A L A B$ Laboratories, Warsaw, Poland
}

\begin{abstract}
Objectives: Copeptin has been reported to play an important role in metabolic response in women with PCOS. However, the optimal cut-off value for detecting subjects with insulin resistance (IR) remains undetermined. We investigated whether copeptin can serve as an indicator of IR and tried to determine the optimal cut-off value of plasma copeptin concentration in detecting subjects with PCOS and IR.

Material and methods: We carried out a case-control study on 158 women with PCOS and HOMA-IR $<2.5,96$ women with PCOS with HOMA-IR $\geq 2.5$, and 70 healthy volunteers. Plasma copeptin, as well as hormonal, biochemical, metabolic, and IR parameters, were measured. To investigate whether copeptin allows IR to be predicted in PCOS, we used logistic regression models and ROC curve analysis.

Results: Median plasma copeptin concentration was the highest in the women with PCOS and HOMA-IR $\geq 2.5$. Logistic regression analysis revealed that copeptin was the strongest predictor of $\mathrm{HOMA} \geq 2.5$ (OR: $53.34 \mathrm{Cl} 7.94-358.23, \mathrm{p}<0.01$ ). Analysis of ROC curves indicated that the cut-off value above $4 \mathrm{pmol} / \mathrm{L}$ of plasma copeptin concentration had high $(99 \%)$ specificity but very low (21\%) sensitivity in diagnosing of IR (AUC 0.607 ( $95 \% \mathrm{Cl} 0.53-0.68$.

Conclusions: Our findings suggest that copeptin is associated with IR in PCOS patients, but due to low sensitivity should not be considered as a marker of IR.
\end{abstract}

Key words: copeptin; PCOS; insulin resistance; metabolic syndrome; AVP

\section{INTRODUCTION}

Polycystic ovary syndrome (PCOS) is a heterogeneous endocrine disease with an estimated occurrence rate of 5-10\% in women of reproductive age [1]. Although metabolic disturbances, such as obesity and insulin resistance (IR), play an important role in the pathogenesis of PCOS, they do not currently form part of the diagnostic criteria. Approximately $50-60 \%$ of women with PCOS are overweight or obese, and IR is found in $50 \%$ of women with PCOS, irrespective of obesity [2]. Women with PCOS have increased cardiometabolic risk - that is, their risk of developing diabetes, hypertension, dyslipidemia, and cardiovascular disorders is greater than the risk within the population as a whole [3]. It is known that IR is the underlying cause for all these car- diovascular and metabolic disorders. Attention has recently been drawn to the role of arginine vasopressin (AVP) in controlling glucose homeostasis, the pathogenesis of $I R$, and the development of diabetes [4-6]. The activation of the hypothalamic-pituitary-adrenal (HPA) axis by AVP under chronic psychosocial stress stimulates the secretion of cortisol by activating $\mathrm{V} 1$ a receptors, which interferes with insulin activity while stimulating glucagon secretion and glycogenolysis [7]. This process subsequently leads to an increase in blood glucose. By activating the $\mathrm{V} 1 \mathrm{~b}$ receptors on chromaffin cells in the adrenal medulla, AVP also increases epinephrine, which contributes to the development of hyperglycemia through glycogenolysis in the liver [7]. Increased AVP, as a result of the resistance of AVP to the V1a receptor, may also contrib- 
ute to IR and to the development of diabetes mellitus (DM) by stimulating the $\mathrm{V} 1 \mathrm{~b}$ receptor [8]. Because of its short half-life and instability, AVP is difficult to measure. Copeptin, the C-terminal fragment of provasopressin, is formed in the same quantities as AVP and, as a result of the processes that activate it, has been found to be a stable and sensitive surrogate marker for AVP release [9].

Recently, it has been demonstrated that plasma copeptin concentrations are elevated in plasma of PCOS patients. Copeptin thus appears to have an important role in metabolic response and in the subsequent development of atherosclerosis in insulin-resistant, hyperandrogenemic PCOS patients $[10,11]$. However, the optimal cut-off value for detecting subjects with metabolic disorders remains undetermined.

\section{Objectives}

The main aim of the study was to investigate whether copeptin can serve as an indicator of IR, and secondly, to determine the optimal cut-off value of plasma copeptin concentration in detecting subjects with PCOS and IR.

\section{MATERIAL AND METHODS}

The study included 254 women with PCOS, aged 18-37 years, and hospitalized in the Department of Reproductive Medicine and Gynecology at the Pomeranian Medical University, Szczecin, and in the Infertility and Reproductive Endocrinology Division of Poznań University of Medical Sciences in the years 2010-2012. Women with PCOS were divided into two groups, depending on the presence of IR: the PCOS(+)IR group consisted of women with a homeostasis model assessment for IR index (HOMA-IR) $\geq 2.5$, while the PCOS(-)IR group included only patients with $\mathrm{HO}$ $M A-I R<2.5$. The study was approved by the Pomeranian Medical University Ethics Committee (No. KB-0012/41/11). The patients were informed of the plan and purpose of the study and gave their written informed consent.

The diagnosis of PCOS was confirmed when at least two of the three diagnostic criteria were present, according to the Rotterdam ESHRE/ASRM-sponsored PCOS Consensus Workshop Group [12]. Clinical hyperandrogenism was defined as a presence of hirsutism (a modified Ferriman-Gallwey score $\geq 8$ ) with or without acne. Metabolic syndrome (MetS) was defined according to the IDF criteria by waist circumference $>80 \mathrm{~cm}$ and at least two of the remaining features: 1. Triglyceride (TG) level: $\geq 150 \mathrm{mg} / \mathrm{dL}$ or specific treatment for this lipid abnormality; 2 . HDL cholesterol level: $<50 \mathrm{mg} / \mathrm{dL}$ or specific treatment for this lipid abnormality; 3 . Systolic blood pressure $\geq 130 \mathrm{mmHg}$ and diastolic blood pressure $\mathrm{BP} \geq 85 \mathrm{mmHg}$, or treatment of previously diagnosed hypertension; 4. Fasting plasma glucose (FPG) $\geq 100 \mathrm{mg} / \mathrm{dL}$ or previously diagnosed type-2 diabetes [13].
The control group consisted of 70 healthy, normally menstruating, age-matched hospital staff and medical students. We included only those subjects who met the following inclusion criteria: eumenorrhea, no medical conditions requiring pharmacological treatment, and no apparent abnormalities in physical examination.

\section{Assessment of clinical variables}

The patients'BMls were calculated from the weight and height measurements, based on the recommendations of the World Health Organization [14]. Waist and hip circumference measurements were also carried out. Blood pressure (BP) was measured using a standard mercury sphygmomanometer with an appropriate cuff size after a resting period of at least $30 \mathrm{~min}$. Hypertension was defined according to the criteria of the Seventh Report of the Joint National Committee on the Prevention, Detection, Evaluation, and Treatment of High Blood Pressure as systolic BP $\geq 140 \mathrm{mmHg}$ and diastolic BP $\geq 90 \mathrm{mmHg}$ [15]. In the early follicular phase, between days 3 and 5 of the cycle, transvaginal ultrasound scans were performed in all patients. Polycystic morphology of ovaries was identified by the sonography according to the established criteria recommended in the literature [12].

\section{Blood sample collection}

Blood sampling was performed in the early follicular phase of the spontaneous or progesterone-induced menstrual cycle (between cycle days 3 and 5), after $12 \mathrm{~h}$ overnight fasting. Serum levels of FSH, $\mathrm{LH}$, estradiol, prolactin, $\mathrm{TSH}, \mathrm{SHBG}$, and insulin were determined by specific electrochemiluminescence assays (automated Elecsys $2010 \mathrm{immu}$ noanalyzer, Roche Diagnostics $\mathrm{GmbH}$ ). The same method, with the use of Cobas 6000 equipment and Roche reagents, was applied to determine total testosterone levels. Levels of serum total cholesterol, high-density lipoprotein (HDL), low-density lipoprotein (LDL), and triglycerides (TG) were determined by semiautomated enzymatic methods (automated Cobas Integra 400Plus analyzer, Roche Diagnostics). The chemiluminescence method was used to determine the levels of androstenedione, DHEA-S, and hs-CRP with the use of Immulite equipment and Siemens reagents). The plasma glucose concentration was determined using the glucose oxidase/peroxidase method. All the basic hormonal and biochemical measurements were performed at the Central Laboratory of University Hospitals.

The free androgen index (FAI) was calculated as the serum testosterone $(\mathrm{nmol} / \mathrm{L}) \times 100 / \mathrm{SHBG}(\mathrm{nmol} / \mathrm{L})$ ratio [16]. IR was diagnosed according to the homeostasis model assessment for IR index (HOMA-IR), and the value was calculated using the following formula: fasting plasma glucose $(\mathrm{mmol} / \mathrm{L}) \times$ fasting serum insulin $(\mathrm{mU} / \mathrm{mL}) / 22.5$. The cut-off value for IR was HOMA-IR $\geq 2.5$ [17]. 


\section{Copeptin assay}

Blood samples were taken into EDTA tubes and centrifuged at $3000 \mathrm{G}$ for $10 \mathrm{~min}$. After that, $500 \mu \mathrm{g}$ of the obtained plasma was separated into a clean test tube with the addition of $5 \mu$ aprotinin. The plasma samples were stored at $-80^{\circ} \mathrm{C}$ until analysis. Copeptin levels were measured in duplicate using competitive enzyme immunoassay (\#EK065-3 Copeptin-Human EIA Kit, Phoenix Pharmaceuticals), carried out in line with the manufacturer's recommendations and using the average values from two measurements. The analytical sensitivity was $0.16 \mathrm{ng} / \mathrm{mL}$. The coefficient of intra-assay repeatability was below $10 \%$, whereas the coefficient of interassay repeatability for this test was $<15 \%$. No cross-reactions were observed in this method.

\section{Statistical analysis}

Since the distributions of most quantitative variables were significantly different from normal, the difference between the groups was tested using the Kruskal-Wallis (test) ANOVA. The Fisher exact test was employed to compare qualitative variables. Where a significant difference was found in the Kruskal-Wallis test, the post hoc test with the Bonferroni adjustment was applied to determine the significant differences. The strength of the correlation between quantitative variables was measured using the Spearman rank correlation coefficient (Rs).

A variety of statistical methods were used to investigate whether copeptin can predict insulin resistance in PCOS. We first used linear and logistic regression models. Univariate linear regression analyses were performed with the copeptin level as the independent variable and HOMA-IR as the dependent variable. Subsequently, these associations were adjusted for covariates that could potentially confound in this association, using multivariable logistic regression models. Multivariable models were built stepwise and the following statistically significant parameters were included: age, $\mathrm{BMI}>25 \mathrm{~kg} / \mathrm{m}^{2}$, SBP, HDL and copeptin $>4.0 \mathrm{pmol} / \mathrm{L}$.

Secondly, a receiver operating characteristic $(\mathrm{ROC})$ analysis was performed to assess the diagnostic value of copeptin as a marker of insulin resistance. P-values $<0.05$ were considered statistically significant. Statistica 10 software (StatSoft, USA) was used for the calculations.

Additionally, to examine the possible correlation between copeptin concentration and the prevalence of MetS, the subjects were stratified by quartiles of copeptin concentration.

\section{RESULTS}

The baseline characteristics of the three study groups are shown in Table 1. The groups differed statistically in terms of anthropometric measurements (BMI, waist circumference, and WHR), chosen hormonal indices (SHBG,
FAI), blood pressure, and HDL and LDL cholesterol. As might be expected, the PCOS(+)IR group had the highest fasting plasma glucose concentration and the highest fasting plasma insulin levels. Plasma copeptin concentration was the highest in the PCOS(+)IR group (median 1.64, interquartile range [IR 0.98-3.27] pmol/L), moderate in the PCOS(-)IR group (1.28 [IR 0.93-2/02] pmol/L), and the lowest (0.97 pmol/L [IR 0.76-1.14]) in the control group $(p=0.014)$. Interestingly, significant differences were found between studied groups regarding the prevalence of MetS, which was present in $37.5 \%$ of PCOS(+)IR, $10.1 \%$ of PCOS(-)IR, and $17.1 \%$ of controls.

Spearman rank correlation coefficient analysis showed no correlation between the plasma copeptin concentration and anthropometric measurements. Plasma copeptin was inversely correlated with FG score $(-0.17 ; \mathrm{p}<0.01)$, and positively correlated with fasting insulin $(0.18 ; p<0.005)$, HOMA-IR $(0.19 ; \mathrm{p}<0.005)$, and systolic and diastolic $\mathrm{BP}(0.14$; $p<0.05 ; 0.17 ; p<0.01)$. Even though the prevalence of MetS was different, we failed to find any correlation between MetS and copeptin ( $p=0.4)$.

In the logistic regression model, the strongest predictor of elevated HOMA-IR $\geq 2.5$ was copeptin (OR: $53.34 \mathrm{Cl}$ 7.94-358.23, $\mathrm{p}<0.01)$. The others were $B M I \geq 25 \mathrm{~m} / \mathrm{kg}^{2}$ (OR: 4.22 Cl: 1.85-9.64, p < 0.01) and SBP (OR: $1.02 \mathrm{Cl}: 1.00$ $1.05, \mathrm{p}=0.03)$. Age (OR: $0.86 \mathrm{Cl}: 0.80-0.93, \mathrm{p}<0.01)$ and HDL (OR: $0.96 \mathrm{Cl}: 0.93-0.98, \mathrm{p}<0.01$ ) were negatively associated with $\mathrm{HOMA}-\mathrm{IR} \geq 2.5$.

The ROC curve assessing the ability of copeptin to distinguish between women with and without IR had an AUC of 0.607 (95\% Cl 0.53-0.68) and a threshold value of $4 \mathrm{mmol} / \mathrm{L}$ or higher to identify insulin-resistant women in the PCOS group (Fig. 1). At this cut-off point, copeptin assay showed very high (99\%) specificity to identify IR in women with PCOS, but low (21\%) sensitivity.

\section{DISCUSSION}

Although IR and its metabolic consequences are not included in the diagnostic criteria of PCOS, it is well known that they play an important role in the pathogenesis of this disease. Copeptin, the C-terminal fragment of provasopressin, is formed in equal quantities as vasopressin (AVP) and, as a result of processes that activate it, has been suggested as a new and promising marker of IR and MetS. Until recently, it was believed that AVP's only role was its effect on the water-electrolyte balance of the body. It has since been shown that, in response to stress factors, AVP and corticotrophin-releasing hormone $(\mathrm{CRH})$ stimulate the secretion of adrenocorticotrophic hormone (ACTH), which increases the secretion of adrenocortical hormones - mainly cortisol and, to a smaller extent, androgens and aldosterone $[18,19]$. Although under physiological condi- 
Table 1. Clinical characteristics of the control, PCOS(-)IR, and PCOS(+)IR groups

\begin{tabular}{|c|c|c|c|c|}
\hline & $\begin{array}{l}\text { Controls } \\
n=70\end{array}$ & $\begin{array}{l}\mathrm{PCOS}(-) \operatorname{IR}[\text { HOMA }<2.5] \\
n=158\end{array}$ & $\begin{array}{l}\operatorname{PCOS}(+) \mid \operatorname{R}[\text { HOMA } \geq 2.5] \\
n=96\end{array}$ & p-value \\
\hline Age [years] & $28(22-30)$ & $28(25-31)$ & $26.5(23-30.5)$ & ns \\
\hline BMI [kg/m2] & $25.3(22.1-28.7)$ & $22.7(20.7-26.5)$ & $28.8(24.8-32)$ & $p<0.001$ \\
\hline Waist circumference $[\mathrm{cm}]$ & $80.5(71-91)$ & $75(69-85)$ & $91(78-101)$ & $p<0.001$ \\
\hline WHR & $0.79(0.74-0.86)$ & $0.78(0.74-0.84)$ & $0.83(0.77-0.88)$ & $p<0.001$ \\
\hline $\mathrm{FSH}[\mathrm{mlU} / \mathrm{mL}]$ & $4.9(3.96-6.25)$ & $5.86(5-6.84)$ & $5.51(4.74-6.77)$ & ns \\
\hline $\mathrm{LH}[\mathrm{mlU} / \mathrm{mL}]$ & $6.04(4.99-7.15)$ & $9.32(5.8-12.7)$ & $7.74(5.39-12.3)$ & ns \\
\hline Estradiol [pg/mL] & $47.1(32.5-70)$ & $46.4(34.4-64.1)$ & $43(33.5-61.6)$ & ns \\
\hline Prolactin [ng/mL] & $15(9.96-19.3)$ & 13.8(9.85-18.8) & $16(10.5-22.1)$ & ns \\
\hline $\mathrm{TSH}[\mu \mathrm{IU} / \mathrm{mL}]$ & $1.63(1.2-2.05)$ & $1.9(1.29-2.45)$ & $1.94(1.31-2.46)$ & ns \\
\hline Testosterone $[\mathrm{ng} / \mathrm{mL}]$ & $0.35(0.25-0.46)$ & $0.46(0.33-0.56)$ & $0.46(0.35-0.61)$ & ns \\
\hline SHBG [nmol/L] & $55.4(39.9-73.4)$ & $45.73(30.5-64.3)$ & $28.3(19.25-50.3)$ & $p<0.001$ \\
\hline FAI & $2.45(1.34-3.57)$ & $3.65(2.18-5.48)$ & $5.43(3.48-9.17)$ & $p<0.001$ \\
\hline DHEA-S [ $\mu \mathrm{g} / \mathrm{dL}]$ & $159(135-181)$ & $247(198-332)$ & $287(212-361)$ & ns \\
\hline FG Score & $0(0-0)$ & $8(4-11)$ & $8(3-10)$ & ns \\
\hline Fasting Glucose [mg/dL] & $85(81-89)$ & $89(83-94)$ & $94.1(88.7-98)$ & - \\
\hline Fasting Insulin $[\mu \mid \mathrm{U} / \mathrm{mL}]$ & $6.83(5.23-9.43)$ & $5.97(4.67-8.08)$ & $14.8(12.9-19.1)$ & - \\
\hline HOMA - IR & $1.42(1.07-2.06)$ & $1.33(0.98-1.83)$ & $3.51(2.96-4.48)$ & - \\
\hline Copeptin [pmol/L] & $0.97(0.76-1.14)$ & $1.28(0.93-2.02)$ & $1.64(0.98-3.27)$ & $p<0.05$ \\
\hline $\mathrm{SBP}[\mathrm{mmHg}]$ & $120(110-130)$ & $120(106-130)$ & $130(120-140)$ & $p<0.001$ \\
\hline $\mathrm{DBP}[\mathrm{mmHg}]$ & $78.5(70-85)$ & $70(60-80)$ & $80(70-85)$ & $p<0.001$ \\
\hline Total Cholesterol [mg/dL] & $165(145-187)$ & 186 (164-209) & $188(168-211)$ & ns \\
\hline HDL Cholesterol [mg/dL] & $59.5(47-69)$ & $66.5(56-78)$ & $51.5(43.1-61.5)$ & $p<0.001$ \\
\hline LDL Cholesterol [mg/dL] & $99(82-116)$ & $102(85.8-120)$ & $110(97-133)$ & $p<0.05$ \\
\hline Triglycerides [mg/dL] & 73 (55-99) & $72(57.9-94.1)$ & $103(72.2-142)$ & $p<0.001$ \\
\hline Metabolic syndrome [\%] & $17.1 \%$ & $10.1 \%$ & $37.5 \%$ & $p<0.001$ \\
\hline hs-CRP [mg/L] & $1.3(0.79-2.59)$ & $1.31(0.69-3.4)$ & $2.05(0.82-4.9)$ & ns \\
\hline
\end{tabular}

Values are expressed as medians (interquartile range); $\mathrm{p}$-values were calculated using the Mann-Whitney post hoc U-test with the Bonferroni adjustment; $\mathrm{p}<0.05$ was considered statistically significant

tions $\mathrm{CRH}$ is a stronger stimulator of ACTH secretion, the synergistic effect of both neurohormones is over 30 times greater than that of $\mathrm{CRH}$ alone. It has been demonstrated that the role of AVP is greater under chronic stress. [19] There is an increase in the number of neurons expressing both CRH and AVP, and the amount of V1bR increases in the pituitary, with reduced $\mathrm{CRH}$ receptor. This effect is resistant to glucocorticoid feedback, suggesting crosstalk between the AVP and hypothalamic-pituitary-adrenal system that could be relevant to insulin resistance and diabetes development $[20,21]$. Research on an animal model involving mice lacking V1aR showed display-impaired glucose tolerance and development of IR with increased AVP levels, whereas mice that lacked V1bR had lower fasting plasma glucose level and increased insulin sensitivity $[22,23]$. Based on these studies, it is assumed that the abnormal effects on V1aR lead to increased AVP levels, which then stimulate
$\mathrm{VbR}$, leading to the development of disorders causing IR and diabetes. Saleem et al. [7] first report the cross-sectional association between high plasma copeptin levels, measures of $\mathrm{IR}$, and the presence of MetS. The researchers reported that plasma copeptin levels correlate significantly with BMI, fasting plasma glucose, insulin level, HOMA-IR, and triglyceride level, and inversely with HDL-cholesterol. Furthermore, the multiple regression analysis that was adjusted for age and sex, plasma copeptin levels in the third and fourth quartiles were significantly associated with higher odds of having MetS. It has been estimated that the activation of the HPA axis by AVP in chromic psychosocial stress may be one of the mediators of its association with IR. It was concluded that such neuroendocrine dysregulation may lead to higher cortisol, decreased energy expenditure, increased appetite and food consumption, increased peripheral vascular resistance, and increased insulin levels. The reported study [7] 


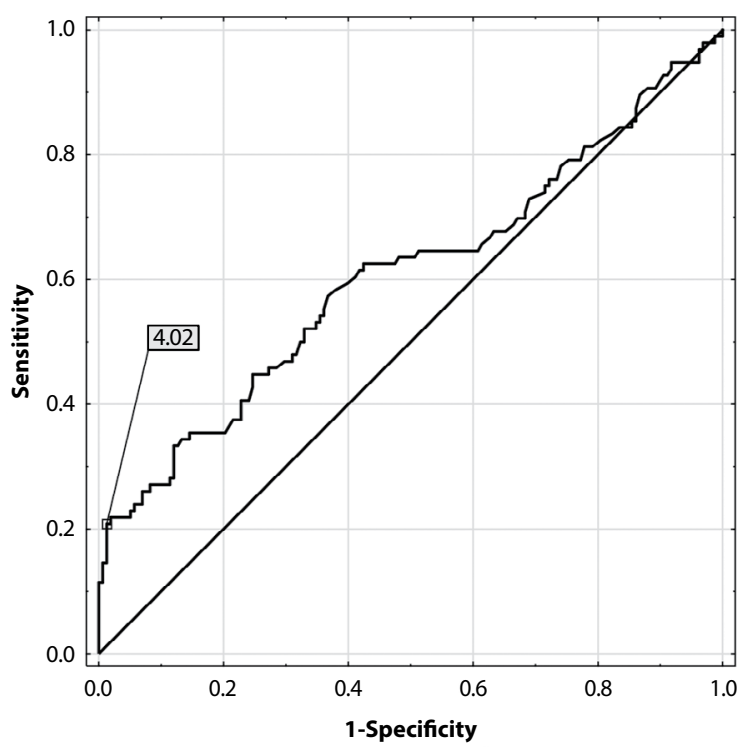

Figure 1. Receiver operating characteristics (ROC) curve assessing the ability of copeptin to distinguish between women with and without IR

included a large cohort that was much older than ours (with a mean age of 59 years) with familial hypertension and a relatively high prevalence of MetS (around 50\%). In agreement with this study, we observed that plasma copeptin levels correlate with IR, but (unlike the results of Saleem et al.) not with full-blown MetS. Interestingly, a cross-sectional study extended the findings of Saleem et al. to a large population-based sample by showing that high copeptin levels are not only associated with the clustering of the MetS components, but that there is also an independent relationship with the core components of the syndrome - i.e., with hypertension and abdominal obesity [24]. Our results suggest that higher plasma copeptin levels are positively correlated with systolic and diastolic BP, but not with full-blown MetS. The differences may be due to significant differences in population characteristics. We speculate that absence of MetS among our subjects is due to the young age. Furthermore, we used different criteria (the IDF criteria) to diagnose MetS; these are more frequently used in Europe. Interestingly, Abbasi et al. have shown that the association of plasma copeptin with the risk of developing DM was stronger in women than in men, which contradicts the previous findings [24, 25]. Researchers have suggested that, because of lower tolerance to changes in AVP levels in women than in men, plasma copeptin both alone and along with the existing biomarkers such as glucose and hs-CRP significantly improved the risk prediction for diabetes in women, but not in men [26-28].

Our study aimed to assess the associations of copeptin concentration with well-known IR markers in women with PCOS. We have shown that plasma copeptin levels mark- edly increased in PCOS patients as compared with healthy women, with special emphasis on the PCOS(+)IR group. This finding is in agreement with the report of Karbek et al. [10], the only study published to date that had considered copeptin in PCOS. Other researchers have reported increased copeptin levels and their positive correlation with fasting insulin, triglyceride, free testosterone, HOMA-IR, and carotid intima media thickness. The above study [10] included only a relatively small group of forty women with PCOS and investigated the correlation between copeptin and the progression of atherosclerosis in PCOS patients. This study demonstrated that copeptin concentrations increase in PCOS patients and are associated with IR. However, the authors did not show whether copeptin can be an indicator of IR, nor did it determine the optimal cut-off value of plasma copeptin in detecting subjects with metabolic disorders. In agreement with the results of Krabek et al., plasma copeptin levels were significantly higher in PCOS than in the non-PCOS group and positively correlated with fasting insulin level, HOMA-IR, and FG score.

In a large prospective cohort study, Enhorning et al. reported that elevated copeptin predicts increased risk of DM independently of established clinical risk factors, including fasting glucose and insulin [8]. The association between copeptin at baseline and the incidence of DM was independent of the incidence of abdominal obesity and vice versa; it is thus possible that AVP independently triggers two different pathways leading to DM and abdominal obesity. Despite this, there is a possibility that the primary elevation of AVP caused by an increase in abdominal fat deposition can lead to the development of DM. Moreover, copeptin may better signal DM susceptibility earlier in the prediabetes state, which would be particularly useful in individuals with normal fasting glucose levels, who are likely to be less closely monitored than patients with impaired fasting glucose $[4,5]$. Because PCOS is a risk factor for the development of type-2 DM, assessing the clinical utility of novel biomarkers such as copeptin would seem to be very important in this group of patients.

Our study showed copeptin to be associated with the risk of increased HOMA IR $\geq 2.5$ in PCOS patients. The ROC analysis implies that copeptin levels above $4 \mathrm{pmol} / \mathrm{L}$ predict IR in PCOS patients with a specificity of $99 \%$, meaning that almost all patients with that level and above have IR, however low sensitivity of $21 \%$ implies that $79 \%$ of IR women with PCOS may also have lower than $4 \mathrm{pmol} / \mathrm{L}$ copeptin levels. Therefore, copeptin cannot serve as a good marker for IR in our study group. Also, AUC value 0.607 reveals that copeptin levels are relatively weak surrogate of IR.

This study has some limitations. First, it was observational, so we could not establish the temporal changes in plasma copeptin, its relationship with preexisting metabolic 
risk factors, or the future development of complications. Second, the number of individuals in the control group was relatively low, and larger studies are needed to confirm our findings. We did not measure either psychosocial stress in the participants or plasma levels of cortisol - the final mediator of a perturbed HPA axis. In our study, we did not use ambulatory BP measurement, which has been shown to be more useful than the causal or office BP measurement [29]. BP, if measured once during the day at a clinical examination, may give overestimated values in patients with white-coat syndrome. Finally, IR was reported based on HOMA-IR values. Although the gold standard for establishing IR is the euglycemic-hyperinsulinemic clamp, this elaborate procedure is not suitable as a screening method. The HOMA-IR calculation correlates very well with the euglycemic-hyperinsulinemic clamp result and is often used as a surrogate marker [30]. However, the cut-off or threshold values for HOMA-IR have not been established. It is well known that the development of IR occurs on a physiological continuum. Our choice of the value 2.5 is based on original HOMA research and previous work with PCOS patients $[17,31,32]$.

\section{CONCLUSIONS}

Our data suggest that copeptin is associated with IR in PCOS patients, but copeptin measurements in plasma have very low sensitivity in detecting IR in this group of patients. Further work is necessary to improve our understanding of the role of AVP in IR and metabolic disorders in PCOS.

\section{Acknowledgements}

This work was supported by the Polish Ministry of Science and Higher Education (Grant MB-322-128/14). The Authors thank Dr. Kornel Chełstowski for help in statistics and preparation of the manuscript.

\section{Conflicts of interest}

The authors report no conflicts of interest.

\section{REFERENCES:}

1. Azziz R, Woods KS, Reyna R, et al. The prevalence and features of the polycystic ovary syndrome in an unselected population. J Clin Endocrinol Metab. 2004; 89(6): 2745-2749, doi: 10.1210/jc.2003-032046, indexed in Pubmed: 15181052.

2. Dunaif A, Segal KR, Futterweit W, et al. Profound peripheral insulin resistance, independent of obesity, in polycystic ovary syndrome. Diabetes. 1989; 38(9): 1165-1174, indexed in Pubmed: 2670645.

3. Wild RA, Carmina E, Diamanti-Kandarakis E, et al. Assessment of cardiovascular risk and prevention of cardiovascular disease in women with the polycystic ovary syndrome: a consensus statement by the Androgen Excess and Polycystic Ovary Syndrome (AE-PCOS) Society. J Clin Endocrinol Metab. 2010; 95(5): 2038-2049, doi: 10.1210/jc.20092724, indexed in Pubmed: 20375205.

4. Muscogiuri G, Barrea L, Annunziata G, et al. Water intake keeps type 2 diabetes away? Focus on copeptin. Endocrine. 2018; 62(2): 292-298, doi: 10.1007/s12020-018-1680-7, indexed in Pubmed: 30027433.
5. Enhörning S, Brunkwall L, Tasevska I, et al. Water supplementation reduces copeptin and plasma glucose in adults with high copeptin: the H2O Metabolism pilot study. J Clin Endocrinol Metab. 2018 [Epub ahead of print], doi: 10.1210/jc.2018-02195, indexed in Pubmed: 30566641.

6. Canivell S, Mohaupt M, Ackermann D, et al. Copeptin and insulin resistance: effect modification by age and $11 \beta$-HSD2 activity in a population-based study. J Endocrinol Invest. 2018; 41(7): 799-808, doi: 10.1007/s40618-017-0807-7, indexed in Pubmed: 29235050.

7. Saleem U, Khaleghi M, Morgenthaler NG, et al. Plasma carboxy-terminal provasopressin (copeptin): a novel marker of insulin resistance and metabolic syndrome. J Clin Endocrinol Metab. 2009; 94(7): 2558-2564, doi: 10.1210/jc.2008-2278, indexed in Pubmed: 19366852.

8. Enhörning S, Wang TJ, Nilsson PM, et al. Plasma copeptin and the risk of diabetes mellitus. Circulation. 2010; 121(19): 2102-2108, doi: 10.1161/CIRCULATIONAHA.109.909663, indexed in Pubmed: 20439785.

9. Holwerda DA. A glycopeptide from the posterior lobe of pig pituitaries. I. Isolation and characterization. Eur J Biochem. 1972; 28(3): 334-339, indexed in Pubmed: 5079944.

10. Karbek B, Ozbek M, Karakose M, et al. Copeptin, a surrogate marker for arginine vasopressin, is associated with cardiovascular risk in patients with polycystic ovary syndrome. J Ovarian Res. 2014; 7:31, doi: 10.1186/17572215-7-31, indexed in Pubmed: 24628831.

11. Taskin Ml, Bulbul E, Adali E, et al. Circulating levels of obestatin and copeptin in obese and nonobese women with polycystic ovary syndrome. Eur J Obstet Gynecol Reprod Biol. 2015; 189: 19-23, doi: 10.1016/j. ejogrb.2015.03.006, indexed in Pubmed: 25837320.

12. Rotterdam ESHRE/ASRM-Sponsored PCOS Consensus W. Revised 2003 consensus on diagnostic criteria and long-term health risks related to polycystic ovary syndrome (PCOS). Fertil Steril. 2004; 81(1): 19-25.

13. The IDF consensus worldwide definition of the metabolic syndrome. Vol. 2005, International Diabetes Federation, 2005.

14. WHO: WHO STEPwise approach to surveillance (STEPS). Geneva: World Health Organization (WHO); 2008.

15. Chobanian AV, Bakris GL, Black HR, et al. Joint National Committee on Prevention, Detection, Evaluation, and Treatment of High Blood Pressure. National Heart, Lung, and Blood Institute, National High Blood Pressure Education Program Coordinating Committee. Seventh report of the Joint National Committee on Prevention, Detection, Evaluation, and Treatment of High Blood Pressure. Hypertension. 2003; 42(6): 1206-1252, doi: 10.1161/01.HYP.0000107251.49515.c2, indexed in Pubmed: 14656957.

16. Vermeulen A, Verdonck L, Kaufman JM. A critical evaluation of simple methods for the estimation of free testosterone in serum. J Clin Endocrinol Metab. 1999; 84(10): 3666-3672, doi: 10.1210/jcem.84.10.6079, indexed in Pubmed: 10523012

17. Matthews DR, Hosker JP, Rudenski AS, et al. Homeostasis model assessment: insulin resistance and beta-cell function from fasting plasma glucose and insulin concentrations in man. Diabetologia. 1985; 28(7): 412-419, indexed in Pubmed: 3899825.

18. Spruce BA, McCulloch AJ, Burd J, et al. The effect of vasopressin infusion on glucose metabolism in man. Clin Endocrinol (Oxf). 1985; 22(4): 463-468, indexed in Pubmed: 3886209.

19. Rabadan-Diehl C, Aguilera G. Glucocorticoids increase vasopressin V1b receptor coupling to phospholipase C. Endocrinology. 1998; 139(7): 3220-3226, doi: 10.1210/endo.139.7.6121, indexed in Pubmed: 9645696.

20. Antoni FA. Vasopressinergic control of pituitary adrenocorticotropin secretion comes of age. Front Neuroendocrinol. 1993; 14(2): 76-122, doi: 10.1006/frne.1993.1004, indexed in Pubmed: 8387436.

21. Scott LV, Dinan TG. Vasopressin and the regulation of hypothalamic-pituitary-adrenal axis function: implications for the pathophysiology of depression. Life Sci. 1998; 62(22): 1985-1998, indexed in Pubmed: 9627097.

22. Aoyagi T, Birumachi Ji, Hiroyama M, et al. Alteration of glucose homeostasis in V1a vasopressin receptor-deficient mice. Endocrinology. 2007; 148(5): 2075-2084, doi: 10.1210/en.2006-1315, indexed in Pubmed: 17303660.

23. Fujiwara $Y$, Hiroyama $M$, Sanbe $A$, et al. Insulin hypersensitivity in mice lacking the V1b vasopressin receptor. J Physiol. 2007; 584(Pt 1): 235-244, doi: 10.1113/jphysiol.2007.136481, indexed in Pubmed: 17673508.

24. Enhörning S, Struck J, Wirfält E, et al. Plasma copeptin, a unifying factor behind the metabolic syndrome. J Clin Endocrinol Metab. 2011; 96(7): E1065-E1072, doi: 10.1210/jc.2010-2981, indexed in Pubmed: 21490073.

25. Abbasi A, Corpeleijn E, Meijer E, et al. Sex differences in the association between plasma copeptin and incident type 2 diabetes: the Prevention 
of Renal and Vascular Endstage Disease (PREVEND) study. Diabetologia. 2012; 55(7): 1963-1970, doi: 10.1007/s00125-012-2545-x, indexed in Pubmed: 22526609.

26. Stachenfeld NS, Splenser AE, Calzone WL, et al. Sex differences in osmotic regulation of AVP and renal sodium handling. J Appl Physiol (1985). 2001; 91(4): 1893-1901, doi: 10.1152/jappl.2001.91.4.1893, indexed in Pubmed: 11568177.

27. Kajantie E, Phillips DIW. The effects of sex and hormonal status on the physiological response to acute psychosocial stress. Psychoneuroendocrinology. 2006; 31(2): 151-178, doi: 10.1016/j.psyneuen.2005.07.002, indexed in Pubmed: 16139959.

28. Rhodes ME, Rubin RT. Functional sex differences ('sexual diergism') of central nervous system cholinergic systems, vasopressin, and hypothalamic-pituitary-adrenal axis activity in mammals: a selective review. Brain Res Brain Res Rev. 1999; 30(2): 135-152, indexed in Pubmed: 10525171.
29. Mancia G, Parati G. Ambulatory Blood Pressure Monitoring and Organ Damage. Hypertension. 2000; 36(5): 894-900, doi: 10.1161/01. hyp.36.5.894.

30. Otten J, Ahrén Bo, Olsson T, et al. Surrogate measures of insulin sensitivity vs the hyperinsulinaemic-euglycaemic clamp: a meta-analysis. Diabetologia. 2014; 57(9): 1781-1788, doi: 10.1007/s00125-014-3285-x, indexed in Pubmed: 24891021.

31. Ascaso JF, Pardo S, Real JT, et al. Diagnosing insulin resistance by simple quantitative methods in subjects with normal glucose metabolism. Diabetes Care. 2003; 26(12): 3320-3325, indexed in Pubmed: 14633821.

32. de Paula Martins W, Santana LF, Nastri CO, et al. Agreement among insulin sensitivity indexes on the diagnosis of insulin resistance in polycystic ovary syndrome and ovulatory women. Eur J Obstet Gynecol Reprod Biol. 2007; 133(2): 203-207, doi: 10.1016/j.ejogrb.2006.10.038, indexed in Pubmed: 17207902. 